\title{
A Novel Recognition Algorithm Based on Optimal Wavelet Packet and Non-Negative Matrix Factorization for Extracting Pathologic Features of Plant Image
}

\author{
Yin Laiwu ${ }^{1}$, Chen Deyun ${ }^{2}$, Li Changcheng ${ }^{2}$ and Chen Dong ${ }^{2}$ \\ ${ }^{1}$ Harbin University of Science and Technology, Harbin, China \\ Jilin Agricultural Science and Technology College, Jinlin, China \\ ${ }^{2}$ Harbin University of Science and Technology, Harbin, China \\ Jilin Agricultural Science and Technology College, Jinlin, China \\ 1yinlaiwu@163.com
}

\begin{abstract}
This paper presents a new recognition algorithm for plant pathology images based on the Non-negative Matrix Factorization, the proposed algorithm is combined with optimal wavelet packet basis to recognize patterns and conduct data encoding in the internet of things oriented intelligent agricultural system. The experimental results show that the performance of the proposed recognition algorithm is far better than those of the principal component analysis and linear discriminant analysis, and the recognition rate are improved, on average, about $14.65 \%$ and $11.18 \%$ higher than the rates of the above algorithms respectively. The presented algorithm is characterized by the fast speed, high calculation accuracy and easy hardware implementation.
\end{abstract}

Keywords: non-negative matrix factorization, optimal wavelet packet basis, plant pathology image, feature extraction

\section{Introduction}

The internet of things is generally referred to the radio frequency identification, but the final internet of things means the image recognition. The compression algorithm is proposed on the basis of the optimal wavelet packet after analyzing the features of data redundancy and visual redundancy existed in the plant pathology images [1]. The method is presented to use the Gabor wavelet for testing the verge of plant root image [2]. The spectrum noise falls into the scope of multiplicative noise, and the method of combining the logarithmic transformation and wavelet transform is put forward on the basis of the theoretical deduction [3], The wavelet soft-threshold de-noising method and the fast Fourier transform (FFT) are used to study the basic features and variation of the electrical signal of aloe leaves under different temperatures [4]. The experiment system of the virtual instrument for collecting the plant electrical signals is designed by using the technology of wavelet soft-threshold denoising method [5]. The continuous Morlet wavelet transform is applied to analyze the variations between the different temperatures, speeds, and humidity data in and out of the corn canopy. The results show that the wavelet transform presents the periodic components included in the variations of micrometeorological factors in and out of the plant canopy [6]. In order to discriminate and recognize the phytoplanktons from different categories, the secondorder low-frequency component of Daubechies wavelet is used to extract the discrete spectrum features of ten phytoplanktons [7]. The method of using the multi-scale wavelet 
transform is presented to test the verge of plant root image [8]. And a wavelet domain semifragile watermarking scheme is put forward for the authentication of color plant disease image [9].

This paper puts forward the combination of the Non-negative Matrix Factorization with the optimal wavelet packet basis to match and recognize the plant pathology images, and the features extraction of the plant pathology image is implemented by using the algorithm to find a mother subspace $\mathrm{W}$ in the spatial domain image to make the pixel points of the basic image of subspace into positive values.

\section{The Principal Theory of Wavelet Packet}

The width of time-frequency window of the basic function of wavelet transform decreases with the decrease of the scale of $\mathrm{J}$, and the width of frequency-domain window will increase accordingly, that is, the domain window of the correspondent wavelet basis function increases with the decrease of the scale.

The distribution (see Figure 1) of small scale against large frequency window and big scale against narrow frequency window is in accordance with the time-frequency characteristics of signals in the natural world, and suitable for analyzing the signals with random scale. However, many problems, in reality, mainly focus on the interested signals at the some certain phases (points) of time or frequency domain, and only the information at the certain point of time and frequency will be necessary for the extraction, so the maximum possible improvement of the resolution ratio of the time-frequency at the interested frequency point, or of the time at the interested time point, should be the aim. And the fixed distribution of timefrequency window of the orthogonal wavelet transform is not the optimal choice under this circumstance, the main reason is that the multi-resolution decomposition of orthogonal wavelet transform only decomposes the $\mathrm{V}$ (scale) space, that is, $V_{0}=V_{1} \oplus W_{1}=V_{2} \oplus W_{2} \oplus W_{j}=\cdots$, but not further decomposes the $\mathrm{W}$ (wavelet) space, and they can be further decomposed with wavelet packet to narrow the frequency spectrum window, which widens with the increase of $\mathrm{j}$ in the process of the orthogonal wavelet transform. And the most suitable time-frequency window or optimal basis can be obtained to analyze the signals [10].

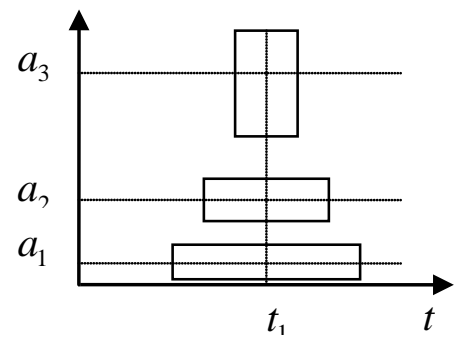

Figure 1. Time-frequency Window of Wavelet Transform

\subsection{The Principal Theory of Wavelet Packet}

2.1.1. The Definition of Wavelet Packet: The two-scale relation between the given orthogonal scaling function $\phi(\mathrm{t})$ and wavelet function $\psi(\mathrm{t})$ is:

$$
\phi(\mathrm{t})=\sqrt{2} \sum_{k} h_{0 k} \phi(2 \mathrm{t}-\mathrm{k}) .
$$




$$
\psi(\mathrm{t})=\sqrt{2} \sum_{k} h_{1 k} \phi(2 \mathrm{t}-\mathrm{k})
$$

The $h_{0 k}$ and $h_{0 k}$ in the expression are the filter coefficients in the analysis of multi-resolution ratio, and the following recursive relations are defined to further expand the two-scale equation:

$$
\begin{array}{r}
\mathrm{W}_{2 \mathrm{n}}(t)=\sqrt{2} \sum_{k \in Z} h_{0 k} W_{n}(2 \mathrm{t}-\mathrm{k}) \\
\mathrm{W}_{2 \mathrm{n}+1}(t)=\sqrt{2} \sum_{\mathrm{k} \in Z} h_{1 k} W_{n}(2 \mathrm{t}-\mathrm{k})
\end{array}
$$

If $n=0$, then $W_{0}(t)=\phi(t), W_{1}(t)=\psi(t)$. The above defined function set $\{\mathrm{wn}(\mathrm{t})\}_{\mathrm{n}} \in \mathrm{Z}$ is the wavelet packet determined by $W_{0}(t)=\phi(t)$. And the wavelet packet $\{\mathrm{wn}(\mathrm{t})\} \mathrm{n} \in \mathrm{Z}$ is a function set containing scaling function $\mathrm{WO}(\mathrm{t})$ and mother wavelet function $\mathrm{W} 1(\mathrm{t})$ with certain connection.

\subsubsection{The Orthogonality of Wavelet Packet}

\section{1) Translation orthogonality}

If the function family $\{\mathrm{wn}(\mathrm{t})\} \mathrm{n} \in \mathrm{Z}$ is set as wavelet packet[11] generated by the scaling function $\mathrm{W} 0(\mathrm{t})=\phi(t)$ of the orthonormal wavelet basis, then they have the translation orthogonality, i.e.

$$
\left\langle w_{n}(t-k) \cdot w_{n}(t-l)\right\rangle=\delta_{k l} \quad k, l \in Z .
$$

\section{2) The orthogonal relation between $w 2 n$ and $w 2 n+1$}

The relation between $w 2 n$ and $w 2 n+1$ is something like the orthogonal relation between $\phi$ and $\psi$. If the function family is the wavelet packet generated by scaling function $\{\mathrm{wn}(\mathrm{t})\} \mathrm{n} \in$ $\mathrm{Z}$ of the orthonormal wavelet basis, then the relation between them is orthogonal:

$$
\left\langle w_{2 n}(t-k) \cdot w_{2 n+1}(t-l)\right\rangle=0 k, l \in Z ; n=0,1,2 \cdots .
$$

\subsection{The Decomposition of Wavelet Packet Subspace}

2.2.1. The Decomposition Process of Wavelet Packet Subspace: The decomposition of wavelet packet can be conducted to obtain the subspace composed with scaling functions and the subspace with wavelet functions. According to the expressions (3) and (4), W0(t) is the scaling function, when $n=0$, and $W 0(t)$ is the wavelet function; when $n=1$, The subspace composed with scaling functions is $\{\mathrm{Vj}\} \mathrm{j} \in \mathrm{Z}$, and the subspace composed with wavelet functions is $\{\mathrm{Wj}\} \mathrm{j} \in \mathrm{Z}$. The following signs are introduced for the discussion of the space composed with wavelet packets:

$$
\begin{cases}U_{j}^{0}=V_{j} & j \in Z \\ U_{j}^{1}=W_{j} & j \in Z\end{cases}
$$

$V_{j}=V_{j+1} \oplus W_{j+1}$ can be obtained from the wavelet multi-resolution ratio analysis, which is presented with the expression (7): 


$$
U_{j}^{0}=U_{j+1}^{0} \oplus U_{j+1}^{1} \quad j \in Z .
$$

When the expression (8) is expanded to the wavelet packet, and

$$
U_{j}^{n}=U_{j+1}^{2 n} \oplus U_{j+1}^{2 n+1} \quad j \in Z, n \in Z^{+} .
$$

The expression (9) is the orthogonal decomposition of the subscript $n$ of the expression (3) and expression (4). $U_{j+1}^{2 n}$ and ${ }^{U_{j+1}^{2 n+1}}$ are the subspaces of ${ }_{j}^{n}$ with ${ }^{U_{j+1}^{2 n}}$ against w2n and ${ }_{j+1}^{2 n+1}$ against ${ }^{W_{2 n+1}}$. ${ }^{2}(R)=\underset{j \in Z}{\oplus} W_{j}$ in the multi-resolution ratio analysis. ${ }^{W_{j}^{n}}$ is expressed as ${ }^{U_{j}^{n}}$ for the convenience of comparison, and the wavelet packet space can be decomposed as:

$$
W_{j}^{n}=U_{j}^{n}=U_{j+1}^{2 n}+U_{j+1}^{2 n+1}
$$

\subsubsection{The Frequency Band of Subspace}

In the subspace of $W j$, the frequency window of frequency band $\mathrm{j}$ is:

$$
H_{J}=\left(\frac{\omega_{0}}{2^{j}}-\frac{\sigma_{0}}{2^{j}}, \frac{\omega_{0}}{2^{j}}+\frac{\sigma_{0}}{2^{j}}\right) \cdot
$$

In the wavelet packet decomposition, the frequency band $\mathrm{j}$ is further decomposed in binary format into $2 \mathrm{k}$ frequency bands; ${ }_{j}^{k, m} \quad m=0,1,2, \cdots, 2^{k}-1$, and all the sub-frequency bands $H_{j}^{k, m}$ compose $H_{j}$, i.e.

$$
\bigcup_{m=0}^{2^{k}-1} H_{j}^{k, m}=H_{j}
$$

From the above expression, the wavelet packet decomposition improves the resolution ratio of the high-frequency components of signals.

\subsection{The Decomposition and Reconstruction of Wavelet Packet}

According to the expression of orthogonal wavelet decomposition of signal $f(t)$

Among them,

$$
P_{J-1} f(t)=P_{j} f(t)+D_{j} f(t) .
$$

The recursive expression of Coefficient $x_{k}^{(j)}$ and $d_{k}^{(j)}$ is:

$$
\left\{\begin{array}{l}
x_{k}^{(j)}=\sum_{n}^{n} h_{0(2 n-k)} x_{n}^{(j-1)} . \\
d_{k}^{(j)}=\sum_{n} h_{1(2 n-k)} d_{n}^{(j-1)}
\end{array}\right.
$$

and the reconstruction expression of the orthogonal wavelet is:

$$
x_{n}^{(j)}=\sum_{k} h_{0(n-2 k)} x_{k}^{(j+1)}+\sum_{k} h_{1(n-2 k)} d_{k}^{(j+1)} \cdot
$$

As $G_{j}^{n} f(t)=G_{j+1}^{2 n} f(t)+G_{j+1}^{2 n+1} f(t)$, the recursive expression of wavelet packet coefficient is:

$$
\left\{\begin{array}{l}
d_{k}^{j+1,2 n}=\sum_{l} h_{0(2 l-k)} d_{l}^{j, n} . \\
d_{k}^{j+1,2 n+1}=\sum_{l} h_{1(2 l-k)} d_{l}^{j, n}
\end{array}\right.
$$

so the reconstruction expression of wavelet packet is: 


$$
d_{l}^{j, n}=\sum_{k} g_{0(l-2 k)} d_{k}^{j+1,2 n}+\sum_{k} g_{1(l-2 k)} d_{k}^{j+1,2 n+1} .
$$

\subsection{The Selection of the Optimal Wavelet Packet Basis}

An optimal wavelet packet basis can be selected according to the different features of signals for analysis to present the signal characteristics [12]. The wavelet packet decomposition of signal $f(t)$ is to project $f(t)$ on the wavelet packet basis with the aim of achieving a series of coefficients $d_{l}^{j, n}$, which are used to describe the features of the signal $\mathrm{f}(\mathrm{t})$, and it is better if the difference between the coefficients is larger, because if only a small number of coefficients $d_{l}^{j, n}$ are large, then these coefficients will represent the features of $\mathrm{f}(\mathrm{t})$, and this kind of wavelet packet basis is evidently better. If the difference between this series of coefficients is not large, it will be difficult to find out the features of $f(t)$, and the correspondent basis can not be the optimal basis. The selection of wavelet packet basis involves two problems, one is what the optimal basis is, and the other is how to select the optimal basis.

In order to describe the properties of the coefficient series $d_{l}^{j, n}$, the cost function of a sequence needs to be defined first, and the basis which can make the cost function minimal is to be found out among the wavelet packet bases in the wavelet library. The minimal cost represents the ultimate efficiency for a given vector, and the basis is the optimal. The cost function can be defined as any real function $\mathrm{M}$ about the sequence, but the additive cost function $\mathrm{M}$ is used most to test the concentration ratio. The concentration ratio here means that the $\mathrm{M}$ will be large when the coefficient is moderate, otherwise, $\mathrm{M}$ will be small when the coefficient difference is large; while the additivity means $M$ is an additive information cost function, if $M(0)=0$ and $M\left(\left\{x_{i}\right\}\right) \sum_{i} M\left(x_{i}\right)$.

Among them, when ${ }^{P_{j}}=\frac{\left|x_{j}\right|^{2}}{\|\left. x\right|^{2}}$, and $\mathrm{P}=0, P \lg P=0$. As the information is half-additive, the additive function ${ }^{\lambda(x)=-\sum_{k}\left|x_{k}\right|^{2} \lg \left|x_{k}\right|^{2}}$ is introduced, and $\mathrm{M}(\mathrm{x})$ can be expressed as:

$$
M(x)=\|x\|^{2} \lambda(x)+\lg \|x\|^{2} .
$$

and both $\lambda(x)$ and $\mathrm{M}(\mathrm{x})$ are the smallest.

If cost function $\mathrm{M}$ has been selected, the concept of optimal basis is defined as the following:

Set $X=\{x j\}$ as a vector in the principal space and $B$ is an orthogonal basis selected from the library. If $\mathrm{Bx}$ is the coefficient with $\mathrm{x}$ set under the basis $\mathrm{B}$, and $\mathrm{M}(\mathrm{Bx})$ is the smallest for $x \in V$, then $\mathrm{B}$ is the optimal basis. The selection method of the optimal basis will be discussed in the following.

If the orthogonal basis library meets the following requirements;

- The subset composed with base vectors which equals the set of non-negative integer $\mathrm{N}$ has the intervals as the following

$$
I_{n K}=\left\lfloor 2^{K} n, 2^{K}(n+1)\right\rfloor \quad K \in Z, n \in N .
$$

- Every basis of the library corresponds to a non-intersect overlap composed with In,k of $\mathrm{N}$.

- If Vn,k equals Vn,k, then $V_{n, k}=V_{2 n, k+1} \oplus V_{2 n+1, k+1}$, and this orthogonal basis library has a binary tree structure. 
If the library is a tree, then the optimal basis can be found through the induction of $\mathrm{k}$. Bn,k is the basis corresponding to the vector In,k, and An,k is the optimal basis of spanBn which constrains $\mathrm{x}$. A single basis exists against $\mathrm{k}=0$, that is, In, 0 is the optimal basis, and An, $0=\mathrm{Bn}, 0$, then the construction of An, $\mathrm{k}$ with $\mathrm{n} \geqq 0$ are as the following:

$$
A_{n, k}=\left\{\begin{array}{cc}
B_{n, k} & M\left(B_{n, k}(x)<\left(A_{2 n, k+1}(x)\right)+M\left(A_{2 n+1, k+1}(x)\right)\right) \\
A_{2 n, k+1} \oplus A_{2 n+1, k+1} & \text { other }
\end{array}\right.
$$

The expression (22) is the optimal orthogonal basis which generates $\mathrm{x}$ for the cost function M.

In the real application, only the frequency-limited decomposition can be implemented on $\mathrm{VN}$. If three-layer decomposition is implemented on $\mathrm{VN}$, the coefficient of signal function $\mathrm{f}(\mathrm{x})$ in each subspace can be calculated through the wavelet packet algorithm, then the cost function value of the coefficient on each layer can be generated through $\mathrm{M}(\mathrm{x})$, and the following steps are implemented to select the optimal basis.

- The numbers representing the information cost are written in the nodes, as shown in Figure 2.

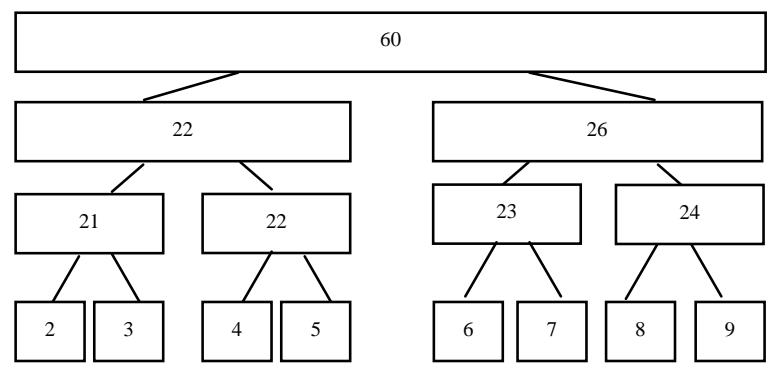

Figure 2. The Information Cost Value of Wavelet Packet

- Starting from the lowest layer, the sign * is marked on the cost function value in each box which represents the node, as shown in Figure 3.

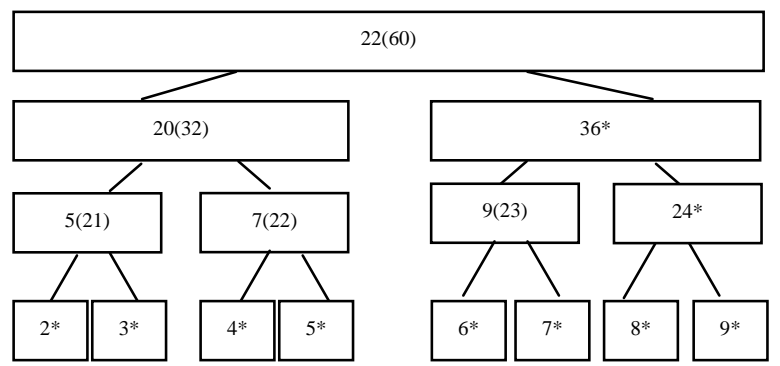

Figure 3. Every Lowest-layer Nodes are Marked

- With the lowest-layer information cost as an initial value, the upper-layer nodes are parents ones, and the lower-layer nodes are son ones. If the information cost of parent node is lower than the son node, then the parent node will be marked, otherwise, unmarked with the value bracketed, and the total value of the two nodes will be written on the outside of the bracket. This procedure is processed until it reaches the top layer, as shown in Figure 4. 


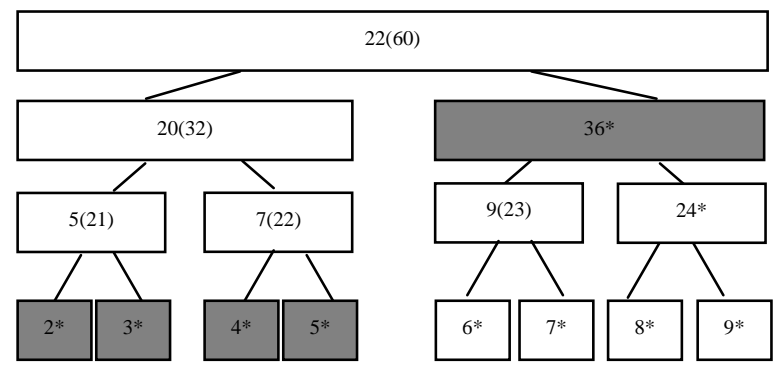

Figure 4. Every Nodes with the Lowest Cost are Marked

- All the nodes are checked, and the top nodes are selected, when it is done, the value of the lower layers will not be considered. All the selected boxes marked with the sign * will compose a set of orthogonal basis, as shown in Figure 5, the nodes of optimal bases in the figure are turned into hatched areas.

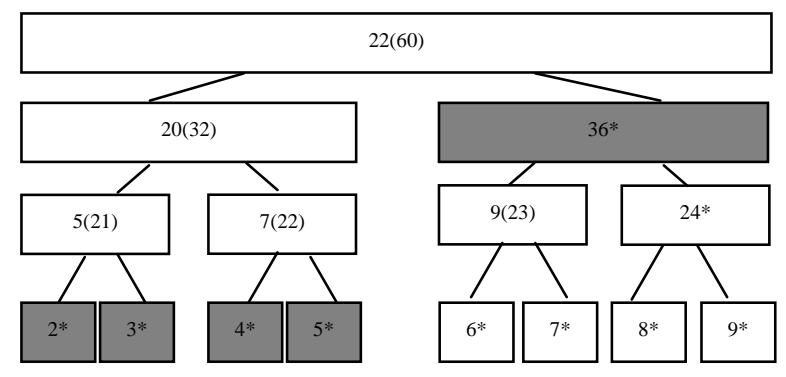

Figure 5. Retain the Top Layer Nodes Marked (optimal basis)

In the view of signal treatment, the process of searching the said optimal basis is using the coefficient as few as possible. And the maximum information can be obtained to achieve the aim of extracting the features.

\section{Features Extraction of the Plant Pathology Image}

In the higher dimensional space, the distribution of plant pathology image is not very contact, which is not good for the classification, and the calculation complexity is very high. Therefore, the efficient method has to be taken for the dimension reduction of image, that is, features extraction. The subspace analysis is generally applied to the image recognition as a algorithm of features extraction, which is characterized by the low cost of calculation and high capacity of description. The principal idea is to find out a linear or non-linear subspace according to a certain performance target to compress the original image into a low dimension subspace with the aim of making the data in the subspace more contact with better extraction of the feature information contained in data.

\subsection{The Steps of Extracting the Plant Pathology Features}

The following two steps are used to extract the plant pathology features through the subspace analysis:

- The training sample are studied to achieve the subspace composed with base vectors and projected in the subspace to obtain the projection coefficients stored in the sample feature library in advance. 
- The sample to be recognized is projected in the subspace to achieve the features of the sample to be recognized.

This paper puts forward an analysis algorithm of the non-negative matrix factorization for feature extraction in comparisons with the principal component analysis and the linear discriminant analysis, and ten images are selected from the plant pathology image library for the training and testing.

\subsection{The Advantages of Extracting the Plant Pathology Features}

As the pixels of the base vectors FCA and LDA can be positive or negative values, both methods lacks the effects of composing the parts into the whole in the visual sense, and this paper proposes NMF as a new algorithm for feature extraction.

The training sample $V=\left\{x_{i}\right\}_{i=1}^{n}$ is composed with n plant pathology images (xi is an $\mathrm{m}$ dimension column vector, which is composed with non-negative gray values of a plant pathology image), and it is decomposed into a product of a non-negative $\mathrm{m} \times \mathrm{r}$ dimension matrix $\mathrm{W}$ and a non-negative $\mathrm{r} \times \mathrm{n}$ dimension matrix $H$ through NMF.

$$
V \approx W H .
$$

The low dimension vector yi is the NMF feature of the sample xi. As shown in Figure 6 [12], the features of the 10 corn pathology images achieved from non-negative matrix factorization algorithm match those from the optimal wavelet packet basis transform, and these ten corn pathology images compose the NMF subspace W.

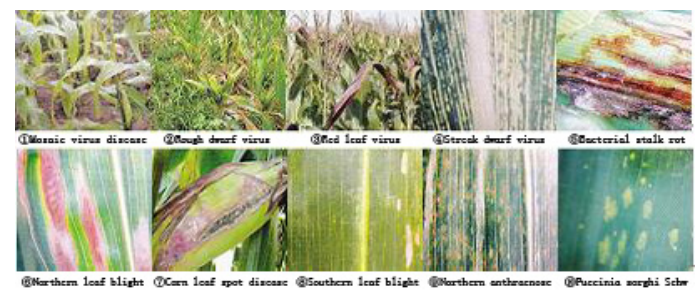

Figure 6. NMF Corn Pathology Images

\section{Simulation Results}

In this paper, the algorithm for extracting the features through the optimal wavelet packet basis transform is implemented on the corn pathology images to achieve the feature extraction of the corn pathology image of non-negative matrix factorization. The non-negative matrix factorization is used to extract the features, and the feature matching is implemented by the optimal wavelet packet basis transform with the results encoded for recognition. Using the optimal wavelet packet basis transform to detect a mutation of maize pathological signals, determine the position of the mutation point. The generation of corn pathological mutation signal as shown in Figure 7, the signal discontinuities due to the latter part of sinusoidal signal in low frequency characteristics into the sinusoidal signal with high frequency characteristic. Application of db1 wavelet decomposition layer 6 to test the first type of discontinuity. The corn pathological details of signal show in Figure 8. 


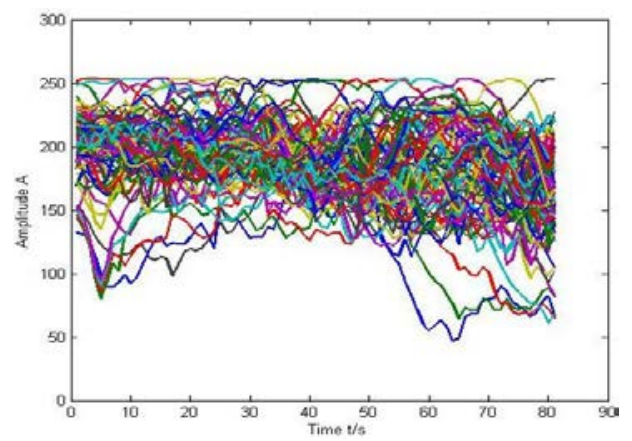

Figure 7. Corn Pathological Mutation Signal Waveform

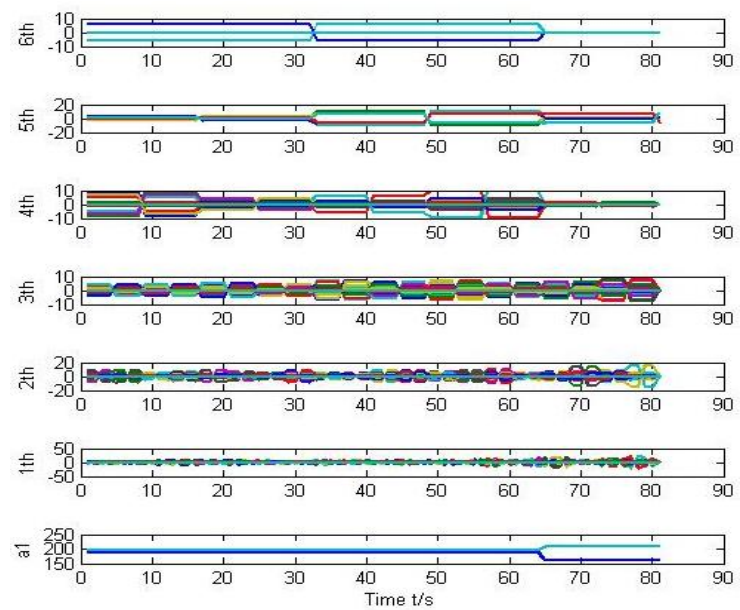

Figure 8. 6th Layers of Db1 Wavelet Decomposition of Corn Pathological Details of Signal

From Figure 7 and Figure 8, the result in the detail signal can clearly show the accurate position of the point of discontinuity. The signal in wavelet decomposition, the fifth layer and the sixth layer detail signal (5th and 6th) discontinuities on signal display quite obvious, because part of the fracture of the signal is contained in the high frequency part, and the approximation signal a1 well to reconstruct the original signal, so that the experimental data tested recognition of pathological signals standard maize code as shown in Table 1.

Table 1. The Results of Recognition Experiment on Com Pathology Library

\begin{tabular}{cccccc}
\hline $\begin{array}{c}\text { Plant } \\
\text { pathology } \\
\text { /category }\end{array}$ & $\begin{array}{c}\text { Mosaic } \\
\text { virus } \\
\text { disease }\end{array}$ & $\begin{array}{c}\text { Rough dwarf } \\
\text { virus disease }\end{array}$ & $\begin{array}{c}\text { Red leaf virus } \\
\text { disease }\end{array}$ & $\begin{array}{c}\text { Bacterial stalk } \\
\text { rot disease }\end{array}$ & $\begin{array}{c}\text { Northern leaf } \\
\text { blight disease }\end{array}$ \\
\hline $\begin{array}{c}\text { Plant } \\
\text { pathology } \\
\text { /category }\end{array}$ & $\begin{array}{c}\text { Corn } \\
\text { leaf } \\
\text { spot } \\
\text { disease }\end{array}$ & $\begin{array}{c}\text { Southern leaf } \\
\text { blight disease }\end{array}$ & $\begin{array}{c}\text { Northern } \\
\text { anthracnose }\end{array}$ & Puccinia sorghi & 0.912 \\
\hline NMF & 0.896 & 0.807 & 0.894 & Streak dwarf \\
virus disease
\end{tabular}


The Table 1 shows the results of recognition encoding experiment on the standard testing images of the 10 corn pathologies.

\section{Conclusion}

In experimental comparisons with other classic recognition algorithms, the pixels of the base vectors of PCA (principal component analysis) and LDA (linear difference analysis) can be positive or negative values, and both methods lack the effects of composing the parts into the whole in the visual sense. On the other hand, the feature matching of optimal wavelet packet basis used in this paper improves the accuracy of the algorithm. The results of contrast experiments of recognition are shown in Table 2.

\section{Table 2. Results of the Contrast Experiment of Different Classic Recognition Algorithm}

\begin{tabular}{llcc}
\hline Classic algorithm & $\begin{array}{c}\text { Average } \\
\text { recognition } \\
\text { ratio }\end{array}$ & $\begin{array}{c}\text { Improvement made by the } \\
\text { proposed scheme against the } \\
\text { listed algorithm }\end{array}$ & $\begin{array}{c}\text { Improvement made by the } \\
\text { proposed scheme against the } \\
\text { listed algorithm (\%) }\end{array}$ \\
\hline PCA & 0.767 & 0.112 & $14.65 \%$ \\
LDA & 0.791 & 0.088 & $11.18 \%$ \\
$\begin{array}{l}\text { The proposed } \\
\text { scheme }\end{array}$ & 0.879 & & \\
\hline
\end{tabular}

The experimental results show that the proposed method in this paper is efficient.

\section{Acknowledgements}

This work is supported by General Program of Jilin Provincial Science and Technology Department (Project No. 201215187).

\section{References}

[1] D. Feng, X. Qiao, X. Du, H. Tian and J. Wen, Journal of Shenyang Agricultural University, vol. 37, no. 274, (2006).

[2] W. Song, L. Wang and Y. Cao, J. Process Automation Instrumentation, vol. 32, no. 24, (2011).

[3] G. Zhou, C. Wang, F. Yang and Y. Li, J. Infrared Millim. Waves, vol. 28, no. 316, (2009).

[4] X. Zhang, N. Yu, G. Xi and X. Meng, Journal of Xi'an University of Technology, vol. 27, no. 411, (2011).

[5] W. Chen, S. Weng, L. Wang, C. Chu and H. Liu, J. Chinese Agricultural Mechanization, vol. 94, (2010).

[6] Z. Jiang, Q. Yu, T. Wang and X. Sun, J. Chinese Journal of Agrometeorology, vol. 25, no. 62, (2004).

[7] F. Zhang, L. Wang, R. Su, Z. Song, X. Wang and C. Zhu, Chinese Journal of Sensors and Actuators, vol. 20, no. 2143, (2007).

[8] W. Song and Z. Wand, J. Development \& Innovation of Machinery \& Electrical Products, vol. 23, no. 135, (2010).

[9] Y. Wang, R. Liu and P. Zhou, Journal of Zhongkai University of Agriculture and Technology, vol. 21, no. 45, (2008).

[10] Q. Meng and W. Zhao, Chinese Journal of Sensors and Actuators, vol. 19, no. 2582, (2006).

[11] O. Mandrikova, I. Solovjev, V. Geppener and D. Klionskiy, J. Pattern Recognition and Image Analysis, vol. 22, no. 323, (2012).

[12] S. K. Hassler, R. M. Lark, A. E. Milne and H. Elsenbeer, European Journal of Soil Science, vol. 62, no. 891, (2011).

[13] H. Shang, F. Wang and R. Shene, "Diagnosis for Plant Diseases”, Color Atlas of Diagnosis and Prevention for Plant Diseases and Insect Pests of Corn, Sorghum \& Millet, JinDun Publishing House, Beijing, China, vol. 6, , (2006), pp. 1-17. 


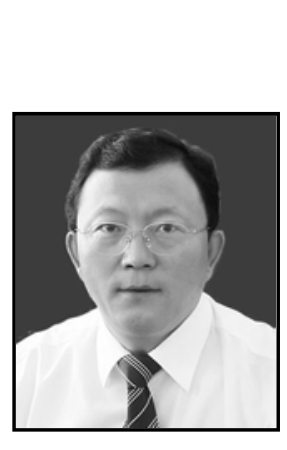

\section{Author}

Laiwu Yin, born in Zhenlai County, Baicheng City, Jilin Province in 1965. He graduated from Jilin University of Finance And Economics as an undergraduate in 2006. At present, He is doing his doctorate in Computer Science and Technology in Harbin University of Science and Technology. His main research direction is agricultural internet of things and intelligent control technology.

He served as the deputy director of Finance Department in Jilin Agriculture Science and Technology College, the Secretary of the Party committee and president of the Institute of Information Engineering Jilin Agriculture Science and Technology College. Now he serves as the director of the Science and Research Division of Jilin Agriculture Science and Technology College, the member of The Hong Kong Institute of International Accounting, vice chairman of "Discovery", research fellow of the Academic Committee Institute of Management Science, the member of National Institutions of Higher Learning computer basic education Society. In recent years, he published five monographs and textbooks, published over 20 papers, three papers of which are SCI, EI indexed. 
International Journal of Signal Processing, Image Processing and Pattern Recognition Vol.6, No.5 (2013) 